The Measurement and Decomposition of Multi-Dimensional Inequality

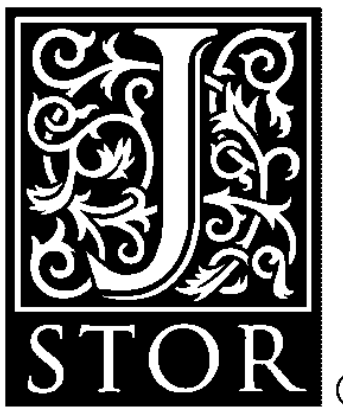

(B) Author(s): Esfandiar Maasoumi

Source: Econometrica, Vol. 54, No. 4, (Jul., 1986), pp. 991-997

Published by: The Econometric Society

Stable URL: http://www.jstor.org/stable/1912849

Accessed: 11/08/2008 13:26

Your use of the JSTOR archive indicates your acceptance of JSTOR's Terms and Conditions of Use, available at http://www.jstor.org/page/info/about/policies/terms.jsp. JSTOR's Terms and Conditions of Use provides, in part, that unless you have obtained prior permission, you may not download an entire issue of a journal or multiple copies of articles, and you may use content in the JSTOR archive only for your personal, non-commercial use.

Please contact the publisher regarding any further use of this work. Publisher contact information may be obtained at http://www.jstor.org/action/showPublisher?publisherCode=econosoc.

Each copy of any part of a JSTOR transmission must contain the same copyright notice that appears on the screen or printed page of such transmission.

JSTOR is a not-for-profit organization founded in 1995 to build trusted digital archives for scholarship. We work with the scholarly community to preserve their work and the materials they rely upon, and to build a common research platform that promotes the discovery and use of these resources. For more information about JSTOR, please contact support@jstor.org. 


\title{
THE MEASUREMENT AND DECOMPOSITION OF MULTI-DIMENSIONAL INEQUALITY
}

\author{
BY ESFANDIAR MAASOUMI ${ }^{1}$
}

\section{INTRODUCTION}

IN THE TRADITIONAL analyses of inequality, individuals are characterized and ordered by a specific set of welfare attributes in which they share. Kolm (1977) formalized the intuitively appealing notion ("fundamentalism") that, the greater the number of attributes considered the better are the assumptions of "anonymity" and "impartiality" in welfare analyses. This notion would argue against the univariate analyses of inequality based traditionally on a single attribute such as income or wealth. Atkinson and Bourguignon (1982) have also described the situations in which the multi-dimensional formulation is either desirable or inevitable. Examples include situations in which "monetization" (through incomes, say) is either problematic or impossible (market prices may not exist, nontraded goods, etc.), or when the "information" given by the objective arguments of individual utility functions is to be augmented, as was argued by Sen (1977).

In the multi-dimensional context, Kolm (1977) analyzed the properties of the social welfare functions (SWF's) and the corresponding ranking of distributions. Atkinson and Bourguignon (1982) analyzed the conditions of first and second-order stochastic dominance of multivariate distributions.

This paper studies a class of evaluative statistics for the measurement of multidimensional inequality and their corresponding SWF's. We derive the properties of the generalized entropy (GE) family of measures. This class contains many of the popular measures, including both of Theil's information measures, and those which are ordinally equivalent to the class of measures proposed by Atkinson (1970).

In our approach an individual (unit etc.) is first represented by a utility-like function of all the attributes received. We refer to this function as the "aggregate" or "summary" attribute. Such a function may also be (and usually is) regarded as the actual utility function of the individual whenever this is appropriate or desirable. ${ }^{2}$ Appealing to a generalized criterion from information theory, we provide an interpretation of this paper's "aggregate" attributes as "ideal" indices or evaluations of individual welfare when the distribution of welfare is the primary concern of analyses (Section 3).

A univariate distribution is thus defined by these aggregates, and the GE measure of inequality therein is referred to as the multi-dimensional or multi-attribute inequality. Multi-attribute inequality is decomposed by the inequality "between" and "within" population subgroups (Section 2). In Section 4, conditions for decomposability by inequality in each attribute are given. The implied SWF's and their properties are also briefly analyzed as are the effects of certain redistributions.

\section{MEASURES OF MULTIVARIATE INEQUALITY}

Let $X_{i f}$ denote the amount of attribute $f=1, \ldots, M$, received by individual (household, country, etc.) $i=1, \ldots, N$. Let $X_{i}=\left(X_{i 1}, \ldots, X_{i M}\right)^{\prime}$ be the $i$ th row of the $N \times M$ matrix

\footnotetext{
${ }^{1}$ Many thanks to Henri Theil for stimulating my interest in the formal aspects of this subject matter, leading to Maasoumi (1979), and to A. Atkinson, J. Foster, A. Shorrocks, the anonymous referees, and Hugo Sonnenschein for many helpful suggestions and criticisms. I retain responsibility for the contents of the paper.

${ }^{2}$ The distinction here is interesting and potentially important. The SWF may be based on the observer's evaluation of individual welfare (the aggregate attribute), or on the individual's own evaluation (the actual utility function). See Kolm (1977, page 3) and Sen (1977).
} 
$X$, and $X^{f}=\left(X_{1 f}, X_{2 f}, \ldots, X_{N f}\right)^{\prime}$. The "summary" or "aggregate" attribute function representing individual welfare is denoted by $S_{i}=h\left(X_{i}\right)$. We measure the relative inequality in $S=\left(S_{1}, \ldots, S_{N}\right)$ by the GE family defined as follows:

$$
I_{\gamma}(S)=\sum_{i=1}^{N} p_{i}\left[\left(S_{i}^{*} / p_{i}\right)^{1+\gamma}-1\right] / \gamma(1+\gamma)
$$

where $S_{i}^{*}=S_{i} / \sum_{j} S_{j}$ and $p_{i}(=1 / N)$ is the $i$ th unit's population share. $I_{1}()$ is ordinally equivalent to the coefficient of variation and the Herfindahl measure, and Theil's first and second measures are, respectively:

$$
\begin{aligned}
& I_{0}(S)=\sum_{i=1}^{N} S_{i}^{*} \log \left(S_{i}^{*} / p_{i}\right), \\
& I_{-1}(S)=\sum_{i=1}^{N} p_{i} \log \left(p_{i} / S_{i}^{*}\right) .
\end{aligned}
$$

For $\gamma<0$ the GE measures are ordinally equivalent to the following measures proposed by Atkinson (1970):

$$
\begin{aligned}
A_{v}(S) & =1-\left[\sum_{i=1}^{N} p_{i}\left(S_{i}^{*} / p_{i}\right)^{1-v}\right]^{1 / 1-v}, \quad v>0, \quad v \neq 1, \\
& =1-\exp \left[\sum_{i} p_{i} \log \left(S_{i}^{*} / p_{i}\right)\right], \quad v=1 .
\end{aligned}
$$

$v=-\gamma$ is referred to as the "degree of relative inequality aversion." As noted by Shorrocks (1980) and Atkinson (1970), the GE and Atkinson measures are homogeneous (in a single attribute), symmetric, and consistent with the Lorenz criterion with respect to $S_{i}$. Useful decomposability properties of $I_{\gamma}($ ) are explicitly given here which separate the "between" and "within" group inequalities for any partitioning of the population. Let there be $G$ groups, $t_{r}, r=1, \ldots, G$, each containing exactly $N_{r}$ individuals, $\sum_{r} N_{r}=N$. Let $P_{r}=N_{r} / N$ and $S_{r}=\sum_{i \in t_{r}} S_{i} / K$ denote the population and the "attribute" shares, respectively, of the $r$ th subgroup, $K=\sum_{i=1}^{N} S_{i}$ and $S .=\left(S_{1}, S_{2}, \ldots, S_{G}.\right)$. Then:

$$
I_{\gamma}(S)=I_{\gamma}(S .)+\sum_{r=1}^{G} P_{r}^{-\gamma} S_{r}^{1+\gamma} I_{\gamma}\left(S^{r}\right)
$$

where $S^{r}$ denotes the $N_{r}$-vector of relative shares $S_{i r}=S_{i} / K S_{r}$., for $i \in t_{r}$, and $I_{\gamma}(S$.) is the multi-dimensional "between" group inequality. The second term of (3) is a weighted sum of "within-group" inequalities, $I_{\gamma}\left(S^{r}\right)$. For Theil's two measures $(\gamma=0,-1)$, however, the familiar weighted averages obtain. In the remainder of this paper our formulae are given for the case of equal population shares-i.e., $p_{i}=1 / N$. The general formulae in (1) should be noted, however, especially when computing the between-group inequalities. In view of this observation, the following equivalence may be verified for the between-group inequality:

$$
I_{\gamma}(S .) \equiv I_{\gamma}\left(\mu_{1} U_{N_{1}}, \mu_{2} U_{N_{2}}, \ldots, \mu_{G} U_{N_{G}}\right) .
$$

The right-hand side is the formula given in Shorrocks (1980), where $\mu_{r}=\sum_{i \in t_{r}} S_{i} / N_{r}$ and $U_{N_{r}}$ is a vector of ones of length $N_{r}$.

In Section 4 we consider the decomposition of $I_{\gamma}(S)$ by attribute inequalities.

\section{REPRESENTATIONS OF INDIVIDUAL WELFARE}

When $S_{i}=h\left(X_{i}\right)$ is interpreted as the actual utility function of an individual, many of the popular (cardinal) specifications in economics can be used in the computations of 
Section 2. Examples include the CES, Cobb-Douglas, linear and log-linear functions and their "flexible" generalizations. When $h($ ) is interpreted as the observer's evaluation of individual welfare, a suitable "index" or "aggregator" function needs to be defined. As in all "index number problems," the intended use of the index affects its "ideal" formulation. Since we are concerned with the study of the multivariate distribution of the $X^{f}$ 's, it is reasonable to require the "index" to have a distribution that is as "close" as possible to this distribution. The use of an "index," $S_{i}$, with the "closest" distribution should entail the introduction of the least amount of distortion in, or divergence from, the distributional "information" given by the $X^{f}$ 's. Information theory provides some of the more general criteria for the measurement of "divergence" or distance between distributions. Here, we use generalized "information functions" which include the logarithm as a special case. This leads us to the notion of $\phi$-entropy; see Burbea and Rao (1982a, 1982b). In turn, we generalize the corresponding, pairwise, criteria of divergence in order to deal with the $M \geqslant 2$ distributions of the $X^{f}$ 's. The result is the following measure of divergence or expected information:

$$
\begin{aligned}
D_{\beta}(S, X ; \alpha) & =\sum_{f=1}^{M} \alpha_{f}\left\{\sum_{j=1}^{N} S_{i}\left[\left(\frac{S_{i}}{X_{i f}}\right)^{\beta}-1\right] / \beta(\beta+1)\right\} \\
& =\sum_{f} \alpha_{f}\left\{\sum_{i} S_{i} \log \left(S_{i} / X_{i f}\right)\right\}, \quad \beta=0, \\
& =\sum_{f} \alpha_{f}\left\{\sum_{i} X_{i f} \log \left(X_{i f} / S_{i}\right)\right\}, \quad \beta=-1 .
\end{aligned}
$$

This criterion is a weighted sum of the pairwise "divergence" terms in \{\} which were shown in Burbea and Rao (1982b) to be generally convex in $S_{i}$. We find the distribution $S=\left(S_{1}, \ldots, S_{N}\right)$ which minimizes $D_{\beta}()$ is as follows:

$$
S_{i} \propto\left[\sum_{f=1}^{M} \delta_{f} X_{i f}^{-\beta}\right]^{-1 / \beta}
$$

where $\delta_{f}=\alpha_{f} / \sum_{f} \alpha_{f}$ (replace by $\alpha_{f}$ when $\sum \alpha_{f}=1$ is used). Clearly the solution is a harmonic mean which includes the weighted geometric mean and the linear functions, popular "indices" in economics. Interestingly, by writing $-\beta=1-(1 / \sigma)$, with $\sigma$ as the constant elasticity of substitution, the above derivation is seen to provide a positive interpretation for many of the popular utility functions in economics, such as the CES, Cobb-Douglas $(\beta=0)$ and the Leontief (linear). [The translog is a second-order approximation to the CES.] Consequently, the formal results of this paper are unaffected whether the $S_{i}$ are regarded as the actual utility functions, or the observer's evaluation of individual welfare obtained according to the "measurement" interpretation given above."

\section{DECOMPOSITION AND THE EFFECTS OF REDISTRIBUTION}

We investigate the GE class of inequality measures when $S_{i}=\left(\sum_{f} \delta_{f} X_{i f}^{-\beta}\right)^{-1 / \beta}$, the most general formulation considered in Section 3. Let $K=\sum_{i} S_{i}, x_{i f}=X_{i f} / T_{f}, T_{f}=\sum_{i} X_{i f}$, $W_{f}=T_{f} / K$, and $S^{*}=\left(S_{1}^{*}, \ldots, S_{N}^{*}\right)$ with $S_{i}^{*}=S_{i} / K$; then:

$$
I_{\gamma}(S)=\frac{1}{N}\left\{\sum_{i} N^{1+\gamma} K^{-(1+\gamma)}\left(\sum_{f} \delta_{f} X_{i f}^{-\beta}\right)^{-(1+\gamma) / \beta}-1\right\} / \gamma(\gamma+1) .
$$

${ }^{3}$ Strictly speaking, the interpretation of $S$ and $X^{f}$ 's as probability distributions requires $\sum_{i} X_{i f}=1=$ $\sum_{i} S_{i}$. This normalization, however, is unnecessary and inconsequential for our derivations. In particular, the same functional form results for the $S_{i}$ which minimizes $D_{\beta}\left(\right.$ ) whether or not $\sum_{i} X_{i f}=1$. When $\beta=-1, D_{-1}()$ is defined when $\sum_{i} S_{i}=\sum_{i}\left(\sum_{f} \alpha_{f} X_{i f}\right)$, and the linear "utility" function, $S_{i}=$ $\sum_{f} \delta_{f} X_{i f}$, minimizes $D_{-1}()$. Log-linear utility obtains if, in $D_{-1}, X_{i f}$ is replaced with $\log X_{i f}$. 
The following proposition gives conditions under which $I_{\gamma}(S)$ may be decomposed into contributions by each attribute $\boldsymbol{X}^{f}$.

Proposition 1: (i) If $1+\gamma=-\beta, I_{\gamma}(S)$ is decomposable as follows:

$$
I_{\gamma}(S)=\sum_{f=1}^{M} \delta_{f} W_{f}^{1+\gamma} I_{\gamma}\left(X^{f}\right)+\left[\sum_{f} \delta_{f} W_{f}^{1+\gamma}-1\right] / \gamma(\gamma+1) ;
$$

(ii) if, in addition, the " marginal" distributions are identical-i.e., $x^{f}=\left(x_{1 f}, x_{2 f}, \ldots, x_{N f}\right)=$ $x^{k}=\left(x_{1 k}, \ldots, x_{N k}\right), \forall f$ and $k$; then we have:

$$
I_{\gamma}(S)=I_{\gamma}\left(X^{f}\right), \quad \text { any } f \in[1, M] .
$$

PROOF: $I_{\gamma}\left(X^{f}\right)$ denotes the GE inequality in the $f$ th attribute distribution according to formula (1), and simple manipulations obtain (7) from (6). In (ii), $x^{f}=x^{k} \Rightarrow S^{*}=x^{f}$, any $f$.

Q.E.D.

PROPOSITION 2: When $1+\gamma=-\beta$, the multi-dimensional extensions of Theil's measures $(\gamma=0,-1)$ are weighted "averages" of attribute contributions:

$$
\begin{aligned}
I_{0}(S) & =\sum_{i} S_{i}^{*} \log N S_{i}^{*} \\
& =\sum_{f} C_{f} I_{0}\left(X^{f}\right)-D_{-1}\left(x, S^{*} ; C_{f}\right),
\end{aligned}
$$

where $C_{f}=\delta_{f} T_{f} / \sum_{k} \delta_{k} T_{k}$, and

$$
\begin{aligned}
I_{-1}(S) & =-\log N-\frac{1}{N} \sum_{i} \log S_{i}^{*} \\
& =\sum_{f} \delta_{f} I_{-1}\left(X^{f}\right)-D_{0}\left(S^{*}, x ; \delta_{f}\right) .
\end{aligned}
$$

ProOF: By direct application of L'Hospital's rule to (7), or using (4), (8a), and (9a). Here $x=\left(x_{i f}\right)$ is the share matrix, and

$$
D_{-1}\left(x, S^{*} ; C_{f}\right)=\sum_{f} C_{f}\left(\sum_{i} x_{i f} \log \frac{x_{i f}}{S_{i}^{*}}\right) \geqslant 0,
$$

where $S_{i}^{*}=\sum_{f} \delta_{f} X_{i f} / K$ and $K=\sum_{f} \delta_{f} T_{f}$ are obtained from (4) at $\beta=-1$,

$$
D_{0}\left(S^{*}, x ; \delta_{f}\right)=\sum_{f} \delta_{f}\left(\sum_{i} S_{i}^{*} \log \frac{S_{i}^{*}}{x_{i f}}\right)=-\log K+\sum_{f} \delta_{f} \log T_{f} \geqslant 0
$$

where $S_{i}^{*}=\prod_{f=1}^{M} X_{i f}^{\delta_{f}} / K$ and $K=\sum_{i}\left(\prod_{f} X_{i f}^{\delta_{f}}\right)$ are obtained from (4) at $\beta=0 . \quad$ Q.E.D.

REMARK 1: Both (10) and (11) are weighted averages of "directional" (KullbackLeibler) measures of expected information, and are nonnegative. It follows from (8)-(11) that, multi-dimensional inequality is no more than the weighted averages of attribute inequalities. This reflects the "substitution" effects between the $X^{f}$ 's.

When individuals are characterized by $S_{i}$, Symmetry (S), Homogeneity (H), and "equality preferring" characteristics of our measures and the corresponding SWF's are established by the literature on univariate inequality; e.g., see Atkinson (1970), Blackorby and Donaldson (1978), Bourguignon (1979), and Shorrocks (1980). 
In the multivariate context, property $\mathrm{H}$ holds in the sense that $I_{\gamma}(S)$ is unchanged if all $X_{i f}$ (i.e., $S_{i}$ ) are multiplied by the same nonnegative constant. $\mathrm{H}$ does not generally hold with respect to each attribute, nor is it necessarily desirable for it to do so. ${ }^{4}$ This "general homogeneity" property is satisfied, however, by Theil's second measure under Proposition 2 (see (9b)), but not by Theil's first measure even though it is only the latter's weights $\left(C_{f}\right.$ 's) that fail to be scale invariant. Property $\mathrm{S}$ here refers to "vector-symmetry"i.e., with respect to vectors $X_{i}, i=1, \ldots, N$, which are the basis for the ordering in the multi-dimensional case. This property implies that $I_{\gamma}()$ of the "welfare matrix" $X$ is the same as that of $\bar{X}=P X$, where $P$ is a permutation matrix.

Let the SWF be $W=F(S)=W(X)$. Any $F$ that is nondecreasing, symmetric, and quasi-concave is also Schur-concave, and hence "equality preferring" (Dasgupta et al. (1973), and Kolm (1977)). In general, however, this property is not particularly meaningful by itself in the multi-dimensional context because it is the $X^{f}$ 's that are the object of policy and directly change with time or across populations. Therefore, we explore the effect on $I_{\gamma}\left(\right.$ ) (or $W(X)$ ) of redistributions in the $X^{f}$ 's. The following Proposition establishes a multi-dimensional version of the Principle of Transfers property.

Let $\bar{X}$ and $X$ be a pair of distributions such that $\bar{X}=B X$, where $B$ is a bistochastic matrix-i.e., $b_{i j} \geqslant 0, \sum_{i}^{N} b_{i j}=1=\sum_{j}^{N} b_{i j}, \forall i$ and $j$. Also, let $\bar{H}$ denote the set of all positive, real valued concave (concave increasing or concave nondecreasing) aggregate functions, $h$, such that $S_{i}=h\left(X_{i}\right)$ and $\bar{S}_{i}=h\left(\bar{X}_{i}\right)$, where $X_{i}$ and $\bar{X}_{i}$ are, respectively, the $i$ th rows of $X$ and $\bar{X}$. Then:

PROPOSITION 3: If $\bar{X}=B X$, where $B$ is a bistochastic matrix, then $I_{\gamma}(\bar{S}) \leqslant I_{\gamma}(S)$ for all $\gamma \in R$ and any $h() \in \bar{H}$ such that $\bar{S}_{i}=h\left(\bar{X}_{i}\right)$ and $S_{i}=h\left(X_{i}\right)$.

Proof: By Kolm (1977, Th. 6), $W(\bar{X}) \geqslant W(X)$ for any $W(X)=F\left(\left\{U\left(X_{i}\right)\right\}\right)$ with concave $U$ and Schur-concave $F$. Since $h(\cdot)$ is concave by assumption and $-I_{\gamma}()$ is Schur-concave (see Shorrocks (1980)), the result holds.

Q.E.D.

REMARK 2: In addition to homotheticity, $F$ may alternatively be symmetric quasiconcave or additive $\left(F=\sum_{i} S_{i}\right)$. In Kolm's Theorem $6, \bar{X}=B X$ is both necessary and sufficient. Hence if $W(\bar{X}) \geqslant W(X)$ for all $W, F$, and $U$ of the above proposition, then $\bar{X}=B X$ for some bistochastic matrix $B$. Strictly speaking, however, this converse result does not fully establish the converse of Proposition 3 as stated since the class $I_{\gamma}($ ) does not contain all Schur-concave $F^{5}$

REMARK 3: Bistochastic transformations are "mean preserving", and amount to several equivalent "averaging" transfers (spreads) which leave the attribute totals ( $T_{f}$ 's) unchanged; e.g., see Kolm (1977). ${ }^{6}$

For concave $S_{i}$, all $I_{\gamma}$ ( ) for which $\gamma \leqslant 0$ certainly satisfy the conditions of Proposition 3. For $\gamma<0$, the SWF's are ordinally equivalent to those of Atkinson's measures which are concave for $v>0$ (Atkinson (1970)). When $\gamma=0$ Theil's first measure obtains with the corresponding SWF proportional to $W=\sum_{i} S_{i} \log \left(\sum_{j} S_{j} / S_{i}\right)$; see Blackorby and Donaldson (1978) and Theil (1967). This is a concave function of $S_{i}$, and thus satisfies

\footnotetext{
${ }^{4}$ For example, doubling $X^{f}$, for a given $f$, affects social welfare, the $S_{i}$, and the ordering theoreof. If $S_{i}$ were defined as an average share-i.e., as a function of $x_{i f}$ instead of $X_{i f}$, the more "general homogeneity" property would have held for all $I_{\gamma}(S)$. In this paper $S_{i}$ is derived in terms of the absolute quantities $\left(X_{i f}\right)$ because actual utility functions are usually defined in terms of $X_{i f}$ rather than the shares $\left(x_{i f}\right)$.

${ }^{5}$ I am grateful to an anonymous referee for bringing this point to my attention and for other helpful criticisms.

${ }^{6}$ If $T_{f}$ 's are not held constant, Proposition 3 may be extended when $B$ transfers are replaced by “equalizing" transfers which satisfy the important properties (1)-(9) of Kolm (1977, page 9). Theorem 9 of the latter provides the basis for this extension.
} 
the conditions of Proposition 3 for concave $S_{i}$. Also, under these conditions the ranking of distributions given by $I_{\gamma}($ ) is consistent with that given by the "Welfare Concentration Curve", a generalization of the Lorenz criterion discussed by Kolm (1977, Th. 7).

The SWF's, $W=F\left(S_{i}\right)$, are clearly individualistic. It is worth noting that, under the decomposability condition of Proposition 1, these SWF's will be both individualistic and specific. Indeed, in Proposition 1 we have $W(X)=H\left[\sum_{i f} F_{i f}\left(X_{i f}\right)\right], H$ and $F_{i f}$ being functions of one variable. Theorem 1 of Kolm (1977) demonstrates that this "additive" form obtains if and only if the SWF is both individualistic and specific. ${ }^{7}$

\section{CONCLUSIONS}

A class of inequality measures has been proposed and analyzed in the multi-attribute context. The choice of these measures was motivated by the axiomatic characterization theorems of Blackorby and Donaldson (1978), Bourguignon (1979), Foster (1983), and Shorrocks (1980) in the univariate case. The propriety and desirability of these axioms deserves closer investigation in the multivariate case. The "measurement" interpretation of individual welfare functions, $S_{i}$, is particularly attractive for attributes which affect individual welfare but cannot reasonably be considered as objective arguments of individual's actual utility functions. With $S_{i}$ so interpreted, the analyses of SWF's based on $S_{i}$ (and evaluative statistics) can incorporate the type of "additional information" which, as Sen (1977) argued, is neglected in the purely welfarist-utilitarian approach. Among some alternative approaches to the measurement of multivariate inequality, Ram (1982) proposes the first principal component (PC) of the matrix $X$ in place of our $S_{i}$. An interpretation of PC is that the linear "aggregate" function, $S_{i}=\sum_{f} \alpha_{f} X_{i f}$, is chosen with $\alpha_{f}$ being the elements of the "first" characteristic vector of the matrix $X^{\prime} X$ (or $x^{\prime} x$, $\left.x=\left(x_{i f}\right)\right)$. Clearly, these weights will be sample-specific and change with $X$. In our approach, on the other hand, the weights given to attributes may be the evaluator's subjective weights. In empirical implementation of our approach, however, $\alpha_{f}$ 's can also be selected on the basis of the PC's (e.g. over all samples of interest, across populations, or over time), but with any desired functional form for $S_{i}$. This is done in Maasoumi and Jeong (1983) and Maasoumi and Nickelsburg (1983). In empirical settings the PC method provides a benchmark which, though rather ad hoc and sample-specific cannot be dismissed lightly, especially where market prices for some attributes do not exist. This is so for the applications in Maasoumi and Nickelsburg (1983) and Maasoumi and Jeong (1983) which utilize data on quality of life and basic needs indices. The treatment of stocks (e.g., wealth) and flows (e.g., income) is another example even when "monetization" of attributes is possible.

Dept. of Economics, Indiana University, Bloomington, IN 47405, U.S.A.

Manuscript received April, 1983; final revision September, 1985.

\section{REFERENCES}

AtKinson, A. B. (1970): “On the Measurement of Inequality," Journal of Economic Theory, II, 244-263.

Atkinson, A. B., AND F. Bourguignon (1982): "The Comparison of Multi-Dimensioned Distributions of Economic Status," Review of Economic Studies, 49, 183-201.

BLACKORBY, C., AND D. DONALDSON (1978): "Measures of Relative Inequality and Their Meaning in Terms of Social Welfare," Journal of Economic Theory, 18, 59-80.

${ }^{7}$ Since $I_{y}($ ) are measures of "relative" inequality and hence homogeneous, the following apparently equivalent situations result in different inequality values: (1) $S W F=H_{1}\left[\sum_{i} U_{i}\right], U_{i}=$ $\log \left(\prod_{f} X_{i f}^{\alpha_{f}}\right)$, and (2) $S W F=H_{2}\left[\sum_{i} \log U_{i}\right], U_{i}=\prod_{f} X_{i f}^{a_{f}}$, where $U_{i}$ are individual utility functions. 
Bourguignon, F. (1979): “Decomposable Income Inequality Measures," Econometrica, 47, 901920.

Burbea, J., AND C. R. RAO (1982a): "Entropy Differential Metric, Distance and Divergence Measures in Probability Spaces: A Unified Approach," Journal of Multivariate Analysis, 12, $575-596$.

(1982b): "On the Convexity of Some Divergence Measures Based on Entropy Functions," IEEE Transactions on Information Theory, IT-28, 489-495.

COWELL, F. A. (1980): "Generalized Entropy and the Measurement of Distributional Change," European Economic Review, 13, 147-159.

Cowell, F. A., AND K. KUGA (1981): "Inequality Measurement: An Axiomatic Approach," European Economic Review, 15, 287-305.

Dasgupta, P., A. Sen, AND D. Starrett (1973): "Notes on the Measurement of Inequality," Journal of Economic Theory, 6, 180-187.

FOSTER, J. E. (1983): "An Axiomatic Characterization of the Theil Measure of Income Inequality," Journal of Economic Theory, 31, 105-121.

Kolm, S.-CH. (1976): "Unequal Inequalities," Journal of Economics Theory, I and II, 12 and 13, 416-442 and 82-111.

- (1977): “Multi-dimensional Egalitarianism," Quarterly Journal of Economics, 91, 1-13.

MAASOUMI, E. (1979): “A Multivariate Index of Inequality Based On Information Theory,” Mimeo, University of Southern California.

(1983): "The Measurement and Decomposition of Multi-Dimensional Inequality," Department of Economics Working Paper 83-5, Indiana University, Bloomington.

MAASOUMI, E., AND G. NickelsburG (1983): "Measuring Joint Inequality in Incomes, Wealth and Schooling and a Decomposition," Department of Economics Working Paper, 83-4, Indiana University, Bloomington.

MAASOUMi, E., in COLlaboration With J-H. JeONG (1983): "The International Distribution of Welfare Attributes: A Multi-Dimensional Analysis of Inequality," Department of Economics, Indiana University, Bloomington.

RAM, R. (1982): "Composite Indices of Physical Quality of Life, Basic Needs Fulfillment, and Income: A 'Principal Component' Representation," Journal of Development Economics, 11, 227-247.

SEN, A. (1977): "On Weights and Measures: Informational Constraints in Social Welfare Analysis," Econometrica, 45, 1539-1572.

SHORROCKS, A. F. (1980): "The Class of Additively Decomposable Inequality Measures," Econometrica, 48, 613-625.

THEIL, H. (1967): Economics and Information Theory. Amsterdam: North-Holland. 